\title{
Fluctuations and Saturation in Diffractive Excitation
}

\author{
Gösta Gustafson* \\ Dept. of Theoretical Physics, Lund University, Lund, Sweden \\ E-mail: gosta.gustafsonethep.lu.se
}

Diffractive excitation is usually described by the Good-Walker formalism for low masses, and by the triple-Regge formalism for high masses. In the Good-Walker formalism the cross section is determined by the fluctuations in the interaction. By taking the fluctuations in the BFKL ladder into account, it is possible to describe both low and high mass excitation in DIS and $p p$ scattering by the Good-Walker mechanism. In high energy $p p$ collisions the fluctuations are strongly suppressed by saturation. This suppression is strongest for central collisions, which implies that diffractive excitation in $p p$ collisions is largest within a ring with radius about $1 \mathrm{fm}$. MC simulations of the Dipole Cascade Model also reproduce the triple-Regge form with a bare pomeron represented by a simple pole with $\alpha(0)=1.21, \alpha^{\prime}=0.2 \mathrm{GeV}^{-2}$, and an almost constant triple-pomeron coupling.

XVIII International Workshop on Deep-Inelastic Scattering and Related Subjects, DIS 2010 April 19-23, 2010

Firenze, Italy

\footnotetext{
*Work done in collaboration with Christoffer Flensburg

$\dagger$ Unfortunately I was not able to come to Florence and present my talk at the conference, due to the ash cloud from the Icelandic vulcano.
} 


\section{Introduction}

Diffractive excitation represents large fractions of the cross sections in $p p$ collisions or DIS. In most analyses of $p p$ collisions low mass excitation is described by the Good-Walker formalism [1], while high mass excitation is described by a triple-Regge formula [2, 3]. In the Good-Walker formalism the state of the incoming projectile is written as a superposition of eigenstates to the $T$ matrix, and the cross section for diffractive excitation is given by the fluctuations in the eigenvalues. In the triple-Regge formulation it is instead determined by the reggeon couplings to the projectile and the target, and a set of triple-reggeon couplings, determined by fits to data (for recent analyses see e.g. refs. [4, 5]). The fluctuations in the pomeron ladder are here not included in the GoodWalker formalism, which therefore limits its application to low masses.

It is, however, well known that the fluctuations in the evolution of a BFKL pomeron are very large [6]. In this talk I will discuss how an analysis of these fluctuations is able to reproduce the triple-regge formulae, with the free parameters determined by fits to the total and elastic cross sections. The fluctuations in the bare pomeron are large both in DIS and in $p p$ collisions, but reduced in the latter by saturation effects. The bare triple-pomeron coupling is also determined. A more extensive presentation of the results is given in ref. [7].

\section{The eikonal approximation and the Good-Walker formalism}

Diffraction, saturation, and multiple interactions are most easily described in impact parameter space. Multiple interactions, which are represented by a convolution in transverse momentum space, correspond to a simple multiplication in transverse coordinate space. If the scattering is driven by absorption into inelastic states $i$, with weights $2 f_{i}$, the elastic amplitude is given by

$$
T=1-e^{-F}, \text { with } F=\sum f_{i} .
$$

For a structureless projectile we find:

$$
d \sigma_{t o t} / d^{2} b \sim\langle 2 T\rangle, \sigma_{e l} / d^{2} b \sim\langle T\rangle^{2}, \sigma_{\text {inel }} / d^{2} b \sim\left\langle 1-e^{-\sum 2 f_{i}}\right\rangle=\sigma_{t o t}-\sigma_{e l} .
$$

If the projectile has an internal structure, the mass eigenstates $\Psi_{k}$ can differ from the eigenstates of diffraction $\Phi_{n}$, which have eigenvalues $T_{n}$. With the notation $\Psi_{k}=\sum_{n} c_{k n} \Phi_{n}$ (with $\Psi_{i n}=$ $\Psi_{1}$ ) the elastic amplitude given by $\left\langle\Psi_{1}|T| \Psi_{1}\right\rangle=\sum c_{1 n}^{2} T_{n}=\langle T\rangle$, while the amplitude for diffractive transition to mass eigenstate $\Psi_{k}$ is given by $\left\langle\Psi_{k}|T| \Psi_{1}\right\rangle=\sum_{n} c_{k n} T_{n} c_{1 n}$. The corresponding cross sections become

$$
\begin{aligned}
d \sigma_{e l} / d^{2} b & \sim\left(\sum c_{1 n}^{2} T_{n}\right)^{2}=\langle T\rangle^{2} \\
d \sigma_{d i f f} / d^{2} b & =\sum_{k}\left\langle\Psi_{1}|T| \Psi_{k}\right\rangle\left\langle\Psi_{k}|T| \Psi_{1}\right\rangle=\left\langle T^{2}\right\rangle .
\end{aligned}
$$

The diffractive cross section here includes elastic scattering. Subtracting this gives the cross section for diffractive excitation, which is determined by the fluctuations in the scattering process:

$$
d \sigma_{\text {diffex }} / d^{2} b=d \sigma_{\text {diff }}-d \sigma_{e l}=\left\langle T^{2}\right\rangle-\langle T\rangle^{2} .
$$

As suggested by Miettinen and Pumplin [8], we will here assume that the diffractive eigenstates correspond to parton cascades, which can come on shell through interaction with the target. 


\section{Dipole cascade models}

Mueller's dipole cascade model $[9,10,11]$ is a formulation of BFKL evolution in transverse coordinate space. Gluon radiation from the colour charge in a parent quark or gluon is screened by the accompanying anticharge in the colour dipole. This suppresses emissions at large transverse separation, which corresponds to the suppression of small $k_{\perp}$ in BFKL. For a dipole $(\mathbf{x}, \mathbf{y})$ the probability per unit rapidity $(Y)$ for emission of a gluon at transverse position $\mathbf{z}$ is given by

$$
\frac{d \mathscr{P}}{d Y}=\frac{\bar{\alpha}}{2 \pi} d^{2} \mathbf{z} \frac{(\mathbf{x}-\mathbf{y})^{2}}{(\mathbf{x}-\mathbf{z})^{2}(\mathbf{z}-\mathbf{y})^{2}}, \quad \text { with } \bar{\alpha}=\frac{3 \alpha_{s}}{\pi} .
$$

The dipole is split into two dipoles, which (in the large $N_{c}$ limit) emit new gluons independently. The result is a cascade, where the number of dipoles grows exponentially with $Y$.

When two cascades collide, a pair of dipoles with coordinates $\left(\mathbf{x}_{i}, \mathbf{y}_{i}\right)$ and $\left(\mathbf{x}_{j}, \mathbf{y}_{j}\right)$ can interact via gluon exchange with the probability $2 f_{i j}$, where

$$
f_{i j}=f\left(\mathbf{x}_{i}, \mathbf{y}_{i} \mid \mathbf{x}_{j}, \mathbf{y}_{j}\right)=\frac{\alpha_{s}^{2}}{8}\left[\log \left(\frac{\left(\mathbf{x}_{i}-\mathbf{y}_{j}\right)^{2}\left(\mathbf{y}_{i}-\mathbf{x}_{j}\right)^{2}}{\left(\mathbf{x}_{i}-\mathbf{x}_{j}\right)^{2}\left(\mathbf{y}_{i}-\mathbf{y}_{j}\right)^{2}}\right)\right]^{2} .
$$

Summing over all dipoles in the cascades then reproduces the LL BFKL result.

The Lund cascade model [12, 13, 14] is a generalisation of Mueller's model, which includes:

- NLL BFKL effects

- Nonlinear effects in the evolution

- Confinement effects

For an incoming virtual photon splitting in a $q \bar{q}$ pair, the initial state wavefunction is determined by perturbative QCD. For an incoming proton we make an ansatz in form of an equilateral triangle of dipoles. After evolution the result is rather insensitive to the exact form of the initial state. The model is also implemented in a MC program DIPSY.

The model reproduces successfully the total and (quasi)elastic cross sections for DIS and $p p$ scattering. The fluctuations in the evolution are large, and the model can also describe diffractive excitation within the Good-Walker formalism, without new parameters beyond those adjusted to the total and elastic cross sections [15]. Here I will discuss the fluctuations, and the effects of saturation, which are very important in $p p$ scattering but less essential in DIS.

\section{Nature of the fluctuations}

\section{$\gamma^{*} p$ collisions}



The distribution in the non-saturated scattering amplitude, $F$, is shown in fig. 1 for different impact parameters. The distribution can be approximately described by a power $\frac{d P}{d F} \approx A F^{-p}$ (with a cutoff for small $F$-values), which is illustrated by 

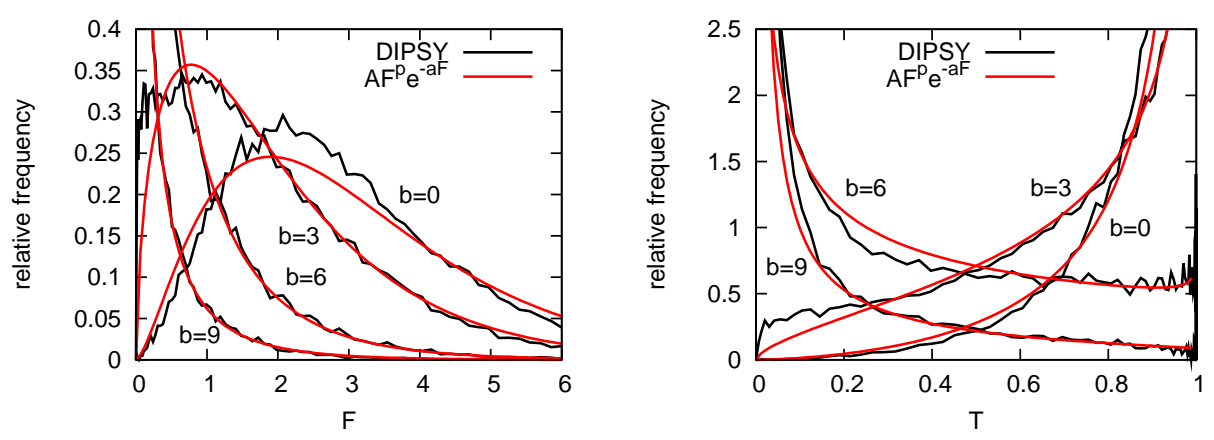

Figure 2: Distribution in the one-pomeron amplitude $(F$, left) and the uniterized amplitude $(T$, right $)$ in $p p$ collisions at $2 \mathrm{TeV}$. Notation as in fig. 1.
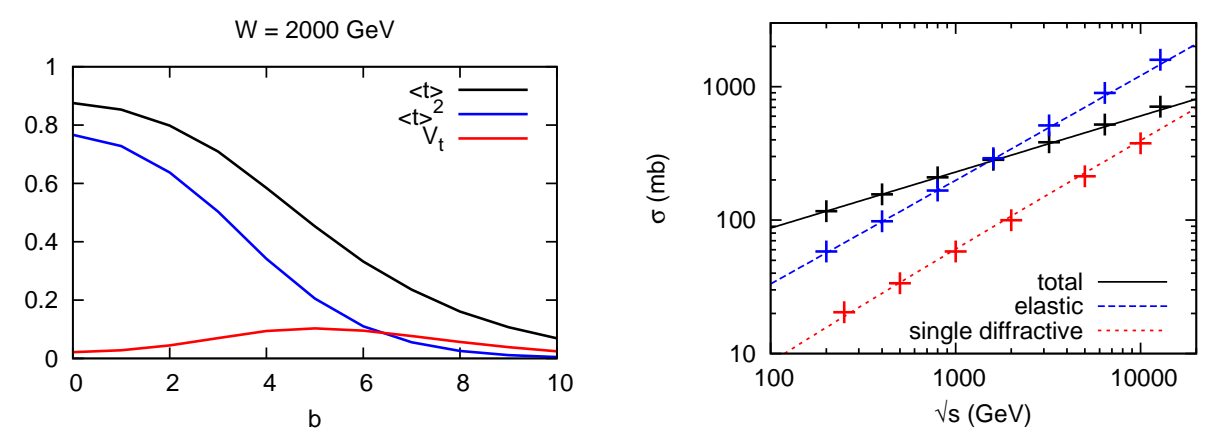

Figure 3: Left: Impact parameter distributions for $\langle T\rangle=\left(d \sigma_{\mathrm{tot}} / d^{2} b\right) / 2,\langle T\rangle^{2}=d \sigma_{\mathrm{el}} / d^{2} b$, and $V_{T}=$ $d \sigma_{\text {diffex }} / d^{2} b$ in $p p$ collisions at $W=2 \mathrm{TeV} . b$ is in units of $\mathrm{GeV}^{-1}$. Right: The total, elastic and single diffractive cross sections in the one-pomeron approximation. The crosses are model calculations and the lines are from a tuned triple-regge parametrisation.

the straight lines in the figure. The width of this distribution is rather large, and the approximation gives the ratio $d \sigma_{\text {diff.ex. }} / d \sigma_{t o t} \approx 1-1 / 2^{2-p}$. The power $p$ is independent of the impact parameter, and therefore this result is also valid for the integrated cross sections. This gives $\sigma_{\text {diff }} / \sigma_{\text {tot }} \sim 0.13$ at $Q^{2}=50 \mathrm{GeV}^{2}$, decreasing for larger $Q^{2}$, but fairly insensitive to the energy $W$.

\section{pp collisions}

In $p p$ scattering the Born amplitude is large, and therefore unitarity effects are important. Fig. 2 shows both the Born amplitude and the unitarized amplitude at $2 \mathrm{TeV}$ for different $b$-values. We see that the width of the Born amplitude is large, and without unitarization the fraction of diffractive excitation would be similar to that for $\gamma^{*} p$ for lower $Q^{2}$-values. (The smooth lines are fits of the form $A F^{p} e^{-a F}$.)

However, the unitarized amplitude is limited by 1 , and the width, and therefore the diffractive excitation, is very much reduced. This is in particular the case for central collisions, where the amplitude approaches the black disc limit. This result corresponds to the effect of enhanced diagrams in the conventional triple-regge approach. As a result factorization is not satisfied when comparing diffractive excitation in DIS and $p p$ scattering. The impact parameter profile is shown in fig. 3. We see that the cross section for diffractive excitation is largest in a ring with radius $b \sim 1 \mathrm{fm}$. 


\section{Relation Good-Walker — Triple-Regge}

Fig. 3 shows also the bare pomeron results for the total, elastic and single diffractive cross sections, without unitarization corrections. We note that these results are very well reproduced by a triple-regge expression with a single pomeron pole, with parameters

$$
\alpha(0)=1.21, \alpha^{\prime}=0.2 \mathrm{GeV}^{-2}, g_{\mathrm{pP}}(t)=\left(5.6 \mathrm{GeV}^{-1}\right) e^{1.9 t}, g_{3 \mathrm{P}}(t)=0.31 \mathrm{GeV}^{-1},
$$

which is shown by the straight lines. We see that without unitarization $\sigma_{e l}$ would be larger than $\sigma_{t o t}$ for $W>1.8 \mathrm{TeV}$.

\section{Summary}

- In the eikonal approximation diffractive excitation is directly determined by the fluctuations in the scattering process.

- The Lund Dipole Cascade Model can describe $p p$ and $\gamma^{*} p$ total, elastic, and diffractive excitation to small and large masses.

- The fluctuations in the cascade evolutions are large.

- Therefore diffractive excitation is large in $\gamma^{*} p$ collisions.

- In $p p$ collisions the fluctuations are large for the Born amplitude, but strongly suppressed by unitarity above $\sim 20 \mathrm{GeV}$.

- Diffractive excitation in $p p$ is a slowly expanding ring in $b$-space with radius $\sim 1 \mathrm{fm}$.

- Neglecting saturation, the dipole model reproduces the triple-Regge result for a bare pomeron, which is a simple pole with $\alpha(0)=1.21$ and $\alpha^{\prime}=0.2 \mathrm{GeV}^{-2}$.

\section{References}

[1] M.L. Good and W.D. Walker, Phys. Rev. 120 (1960) 1857.

[2] A.H. Mueller, Phys. Rev. D2 (1970) 2963.

[3] C.E. DeTar et al., Phys. Rev. Lett. 26 (1971) 675.

[4] M.G. Ryskin, A.D. Martin, and V.A. Khoze, Eur. Phys. J. C60 (2009) 249 [arXiv:0812.2407]

[5] A.B. Kaidalov and M.G. Poghosyan, arXiv:0909.5156.

[6] A.H. Mueller and G.P. Salam, Nucl. Phys. B475 (1996) 293 [hep-ph/9605302].

[7] C. Flensburg and G. Gustafson, arXive: 1004.5502

[8] H.I. Miettinen and J. Pumplin, Phys. Rev. D18 (1978) 1696.

[9] A.H. Mueller, Nucl. Phys. B415 (1994) 373.

[10] A.H. Mueller and B. Patel, Nucl. Phys. B425 (1994) 471 [hep-ph/9403256].

[11] A.H. Mueller, Nucl. Phys. B437 (1995) 107 [hep-ph/9408245].

[12] E. Avsar, G. Gustafson, and L. Lönnblad, JHEP 07 (2005) 062 [hep-ph/0503181].

[13] E. Avsar, G. Gustafson, and L. Lönnblad, JHEP 01 (2007) 012 [hep-ph/0610157].

[14] C. Flensburg, G. Gustafson, and L. Lönnblad, Eur. Phys. J. C60 (2009) 233, [arXiv:0807.0325].

[15] E. Avsar, G. Gustafson, and L. Lönnblad, JHEP 12 (2007) 012 [arXiv:0709.1368]. 